\title{
Atendimentos por causas acidentais em serviços públicos de emergência - Teresina, Piauí - 2009
}

\author{
Medical care due to accidental causes \\ in emergency public services - Teresina, Piauí - 2009
}

\author{
Ana Amélia Galas Pedrosa ${ }^{1}$ \\ Márcio Dênis Medeiros Mascarenhas ${ }^{2}$ \\ Elaine Monteiro da Costa ${ }^{3}$ \\ Liana Pereira Cronemberger ${ }^{1}$
}

${ }^{1}$ Fundação Municipal de Saúde (FMS), Teresina-PI. Rua Gov. Raimundo Artur de Vasconcelos n 3015 , Aeroporto. 64006-080 Teresina PI. anaameliapedrosa@gmail.com ${ }^{2}$ Programa de PósGraduação em Ciências Médicas, Universidade Estadual de Campinas (UNICAMP).

${ }^{3}$ Programa de PósGraduação em Ciências e Saúde, Universidade Federal do Piauí (UFPI).

\begin{abstract}
Accidents are a major issue in the public health agenda and accidents from external causes represent a current challenge for public policies. The scope of this article is to describe the characteristics of emergency care grouped in the "other accidents" category of the "VIVA" 2009 survey, in the public services of Teresina-Piaui. Data was collected from standard forms entered into Epi Info 3.5.1 and analyzed in Stata 11 (module svy). Characteristics relating to the victims, the occurrence and care were compared according to age group and the type of accident using the Pearson chi-square test. Of the total of 2,061 external cause accidents, 677 (32.9\%) were due to "other accidents," of which 202 (29.8\%) involved wounds from sharp cutting objects, 172 (25.4\%) with objects falling on the person or the person hitting an object, 111 (16.4\%) with unknown objects and $70(10.3 \%)$ with accidents involving animals. Accidents in the household with injuries located on the torso leading to hospitalization were greater in patients $<20$ years $(p<0,005)$. Important differences were identified in the pattern of the occurrence among the types of accidents. The magnitude of these events suggests the need for programs that reduce vulnerability to accidents, linking health promotion and prevention actions.
\end{abstract}

Key words Health vigilance, External causes, Accidents, Emergency care
Resumo As causas externas representam atual desafio para as políticas públicas, destacando os acidentes na agenda pública de saúde. Este artigo objetiva descrever as características dos atendimentos de emergência agrupados na categoria "outros acidentes" do Inquérito VIVA 2009, em serviços públicos de Teresina, Piauí. Os dados foram coletados em formulário padronizado, digitados no Epi Info 3.5.1 e analisados no Stata 11 (módulo svy). Compararam-se as características relativas à vítima, ocorrência e atendimento, segundo faixa etária e tipo de acidente por meio do teste do qui-quadrado de Pearson. Do total de 2.061 atendimentos por causas externas, 677 (32,9\%) foram devidos a "outros acidentes", dos quais 202 (29,8\%) foram por ferimento com objeto perfurocortante, $172(25,4 \%)$ por queda de objeto sobre pessoa/choque de pessoas contra objeto, 111 $(16,4 \%)$ por corpo estranho e 70 (10,3\%) de acidentes com animais. Acidentes no domicílio, com lesões localizadas no tronco e com evolução para internação foi mais frequentes em pacientes $<20$ anos ( $p<0,005)$. Identificaram-se diferenças significativas no padrão de ocorrência entre os tipos de acidentes. A magnitude desses eventos sugere a necessidade de programas que reduzam a vulnerabilidade aos acidentes, articulando ações de prevenção e promoção da saúde.

Palavras-chave Vigilância em Saúde, Causas externas, Acidentes, Atendimento de emergência 


\section{Introdução}

As causas externas (acidentes e violências) representam, na atualidade, um problema de saúde pública de dimensões mundiais e seu enfrentamento, um desafio para as políticas públicas. Os acidentes e a consequente demanda por atendimento no setor saúde têm se tornado, ao longo dos últimos anos, um problema que ocupa lugar de destaque na agenda pública de saúde ${ }^{1,2}$.

Os acidentes são entendidos como eventos fortuitos, não intencionais, muitas vezes evitáveis, responsáveis por óbitos e lesões de diferente gravidade. Podem ocorrer no ambiente doméstico ou nos sociais como o do trabalho, de circulação de pessoas e veículos, escola, esporte e lazer². Apesar disso, comportam um grau de imprecisão, exatamente pela dificuldade em se estabelecer o caráter de intencionalidade ou fatalidade ${ }^{3}$.

São considerados, ainda, como problema universal, pois independem do estágio de desenvolvimento dos países. Acontecem nos países desenvolvidos, que apresentam determinados padrões de urbanização, motorização e circulação de pessoas. E ocorrem, também, nos países em desenvolvimento, que revelam aspectos de natureza social, alterações socioeconômicas, equipamento industrial obsoleto, condições ambientais de moradia desfavoráveis, composição do núcleo familiar e nível de escolaridade relacionados à ocorrência de acidentes ${ }^{4,5}$.

As estatísticas de morbidade e mortalidade para os acidentes (exceto os de transporte) fazem, quase sempre, menção às características de ocorrência no trabalho ou no ambiente doméstico, aos ciclos de vida e à gravidade da lesão, sobretudo aos acidentes de maior gravidade ou fatais $^{6-12}$. No entanto, a incidência elevada de lesões não fatais em decorrência de acidentes com instrumentos perfurocortantes, penetração de corpo estranho em orifícios do corpo, queda de objeto sobre pessoa, choque pessoas contra objeto, acidente com animais dentre outros (sufocação/engasgamento, afogamento, ferimento por arma de fogo), demandam atendimento especializado e determinam muitas vezes importantes sequelas ${ }^{10-14}$.

Apesar da elevada ocorrência desses tipos de acidentes, ainda é escassa a produção de análise acerca de sua caracterização epidemiológica, o que limita a utilização das informações no âmbito do setor saúde como ferramenta para a formulação e a gestão de políticas de saúde específicas para o enfrentamento da problemática, revelando, assim, alguns desafios a serem enfrenta- dos. Entre eles, pode-se citar a consolidação das informações detalhadas nos prontuários, quando o atendimento evolui para internação, a produção de registros oportunos para os casos com liberação imediata pós-atendimento e outras informações relevantes, para que as ações decorrentes da política sejam efetivas ${ }^{9,15-18}$.

Para o monitoramento das causas externas no Brasil, o Ministério da Saúde conta com dados do Sistema de Informações sobre Mortalidade (SIM), Sistema de Informações Hospitalares do SUS (SIH/SUS) e, a partir de 2006, com os dados do Sistema de Vigilância de Violências e Acidentes (VIVA) formado por dois componentes - VIVA Continuo e VIVA Inquérito ${ }^{1,19-21}$. O objetivo do VIVA Inquérito é caracterizar as vítimas de violência e acidentes atendidas em serviços de urgência e emergência, por meio de um levantamento de dados que ocorra a cada dois anos. Com essa proposição, as informações epidemiológicas sobre o comportamento desses agravos subsidiam ações de enfrentamento dos determinantes e condicionantes das causas externas $^{1,20,21}$.

As informações atualmente disponíveis sobre atendimentos de emergência por causas acidentais têm sido limitadas a analisar o padrão epidemiológico de quedas ${ }^{22}$, acidentes de transporte $^{23}$ e queimaduras ${ }^{24}$, revelando uma lacuna no conhecimento mais detalhado sobre as demais causas acidentais ("outros acidentes"), apesar da elevada frequência de casos dentre os atendimentos de emergência, suscitando questionamentos cujas respostas possam contribuir para o direcionamento de políticas de prevenção dos acidentes e promoção da saúde.

Este trabalho objetiva descrever as características dos atendimentos de emergência classificados como "outros acidentes", quais sejam ferimentos por objetos perfurocortantes, queda de objeto sobre pessoa/choque de pessoas contra objeto, penetração de corpo estranho em orifícios do corpo, acidentes com animais, intoxicação/envenenamento, sufocação/engasgamento, afogamento, ferimento por arma de fogo, segundo aspectos demográficos, da ocorrência e dos atendimentos do VIVA Inquérito 2009, em Teresina-Piauí.

\section{Métodos}

O Sistema de Vigilância de Acidentes e Violência (VIVA) no Sistema Único de Saúde (SUS) possui o componente Inquérito (VIVA Inquérito), que 
a cada dois anos, é realizado nas capitais, Distrito Federal e alguns municípios selecionados. Nacionalmente, tem a coordenação do Ministério da Saúde e, em Teresina, esteve sob a responsabilidade da Fundação Municipal de Saúde, órgão gestor do SUS no município.

O estudo realizado é de corte transversal. Seus dados foram coletados no período de 30 dias consecutivos, em outubro de 2009, em 16 turnos de 12 horas por cada unidade, selecionados por sorteio probabilístico em todos os serviços de urgência e emergência $(n=9)^{1}$, correspondendo ao universo de serviços públicos da gestão municipal. Os serviços selecionados atenderam aos critérios de número de atendimentos por causas externas, complexidade e resolutividade do serviço, além da consulta ao Cadastro Nacional de Estabelecimento de Saúde (CNES) e aos registros do SIH-SUS ${ }^{1}$. Foram excluídos os serviços privados.

Os atendimentos por acidentes e violência nos hospitais selecionados compuseram a população do estudo. Realizou-se sorteio prévio para definição dos turnos por meio de amostragem por conglomerado em estágio único, com o objetivo de atingir uma amostra de 1.500 entrevistas, assumindo coeficiente de variação inferior a $30 \%$ e o erro padrão menor que três. O turno foi considerado a unidade primária de amostragem (UPA). Deste modo, todos os atendimentos por causas externas, ocorridos no turno sorteado, foram incluídos na amostra. Os dados foram coletados em formulário padronizado com variáveis organizadas em blocos contendo: dados gerais da notificação, da pessoa atendida, da residência, da ocorrência, tipo de acidentes e violência, natureza da lesão corporal, parte do corpo atingida e evolução dos casos ${ }^{1}$.

As entrevistas foram realizadas por acadêmicos dos cursos de medicina e enfermagem submetidos a processo seletivo e a capacitação de 20 horas-aula sobre vigilância de causas externas e metodologia do VIVA Inquérito. A capacitação foi conduzida pela equipe local responsável pelo inquérito, seguindo orientações acerca das atribuições dos entrevistadores, supervisores e coordenadores, definições e demais procedimentos para coleta de dados, de acordo com o Manual do Entrevistador, elaborado pela coordenação nacional do inquérito ${ }^{25}$.

Posteriormente, os dados foram digitados no programa Epi Info 3.5.1, submetidos à análise de consistência e verificação de duplicidade por meio do programa Link Plus versão 2.0. As análises estatísticas foram realizadas no programa Stata versão 11, por meio do módulo "svy", apropria- do para obter estimativas dos parâmetros populacionais não viciadas com dados provenientes de planos de amostragem complexos.

A presente análise restringiu-se aos acidentes com instrumento perfurocortante, queda de objeto sobre pessoa/choque de pessoa contra objeto, penetração de corpo estranho, acidente com animais e, em algumas situações, os demais atendimentos de maneira agrupada, incluindo: sufocação/engasgamento, afogamento, envenenamento/intoxicação, ferimento por arma de fogo, entorse/torção e esmagamento.

Esses eventos são organizados na Classificação Estatística Internacional de Doenças e Problemas Relacionados à Saúde 10a revisão (CID 10) ${ }^{26}$ nos seguintes grupamentos: exposição a forças mecânicas inanimadas (W20-W49); exposição a forças mecânicas animadas (W50-W64); afogamento e submersão acidentais (W65-W74); outros riscos acidentais à respiração (W75-W84); contato com animais e plantas venenosas (X20X29); envenenamento (intoxicação) acidental por exposição a substâncias nocivas (X40-X49); e exposição acidental a outros fatores e aos não especificados (X58-X59). Para a agregação das categorias queda de objeto sobre pessoa (W20) e choque de pessoa contra objeto (W22) considerouse a semelhança da exposição dessas ocorrências.

As análises foram feitas para o conjunto dos atendimentos dos nove serviços incluídos na pesquisa, segundo variáveis do indivíduo/vítima (sexo, faixa etária, escolaridade raça/cor da pele), da ocorrência (tipo e local da ocorrência, meio de locomoção utilizado para o hospital, evento relacionado ao trabalho, declaração de consumo de bebida alcoólica) e dados do atendimento propriamente dito (parte do corpo atingida, evolução, período e dia de atendimento).

A comparação das características considerou a faixa etária divida em dois grupos: menores de 20 anos (crianças e adolescentes) e 20 e mais anos (adultos e idosos). Apresentou-se, também, as características dos atendimentos comparandoas por tipo de evento, excluindo-se envenenamento/intoxicação, esmagamento, entorse/torção e outros por apresentarem menor magnitude. As diferenças entre as frequências foram estimadas por meio do teste do qui-quadrado de Pearson, considerando-se como estatisticamente significante o valor de p inferior a 0,05 .

$\mathrm{O}$ projeto de pesquisa foi aprovado pela Comissão Nacional de Ética em Pesquisa do Ministério da Saúde, conforme recomendação da Resolução No 196/199627, do Conselho Nacional de Saúde. Tendo em vista que a pesquisa foi uma 
ação específica de vigilância epidemiológica de âmbito nacional, não houve termo de consentimento livre e esclarecido, embora tenha havido consentimento verbal, obtido pelo paciente ou responsável.

\section{Resultados}

Durante o Inquérito VIVA 2009, no município de Teresina, foram identificados 2.061 atendimentos de emergência por acidentes e violência, sendo $1.815(88,1 \%)$ por acidentes e $246(11,9 \%)$ por violência. Os atendimentos por outras cau- sas acidentais representaram $32,9 \%$ do total, seguidos por acidentes de transporte $(31,5 \%)$, quedas $(22,6 \%)$ e queimaduras $(<1,0 \%)$. As agressões corresponderam a $10,6 \%$, onde se inclui maus tratos e intervenção por agentes públicos, enquanto as lesões autoprovocadas representaram $1,3 \%$ de todos os atendimentos por causas externas (Figura 1). Considerando apenas os atendimentos de emergência por outras causas acidentais, predominaram atendimentos por ferimento com objeto perfurocortante $(29,8 \%)$, queda de objeto sobre pessoa/choque de pessoa contra objeto $(25,4 \%)$, corpo estranho $(16,4 \%)$ e acidentes com animais (10,3\%) (Figura 2).

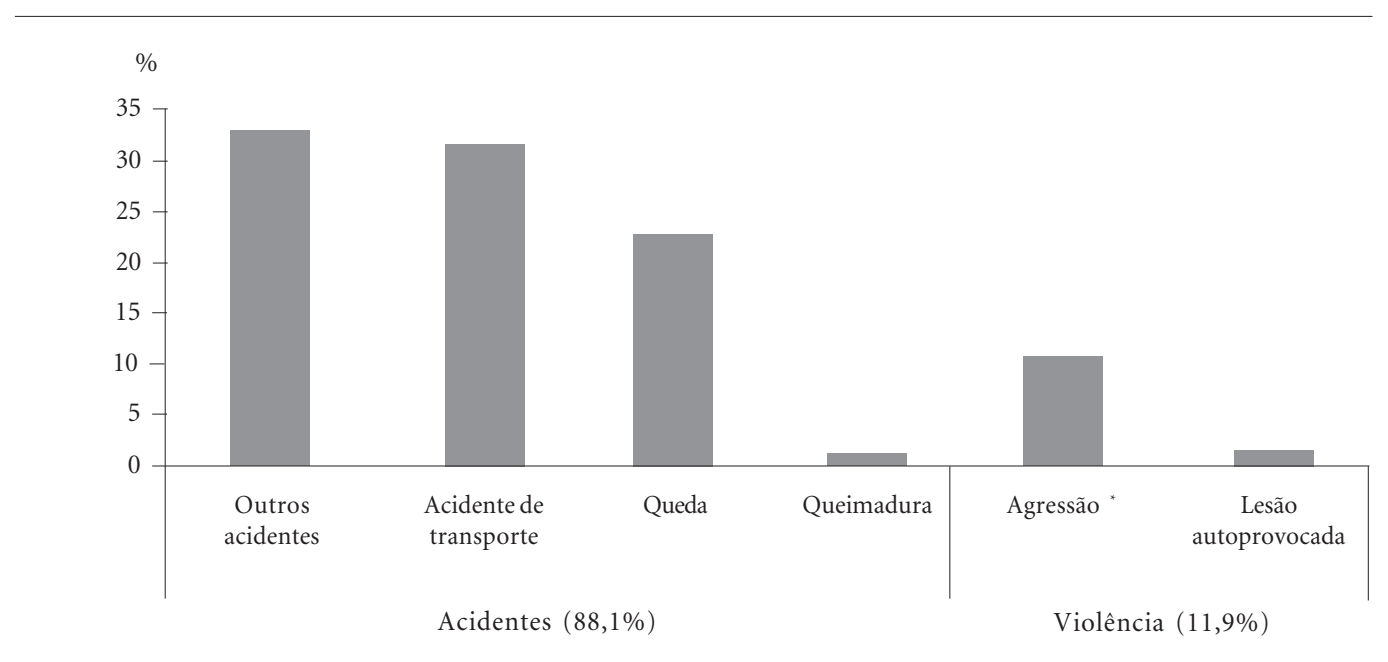

Figura 1. Distribuição proporcional de atendimento de emergência por acidentes e violência segundo tipo de ocorrência. Teresina-PI, 2009.

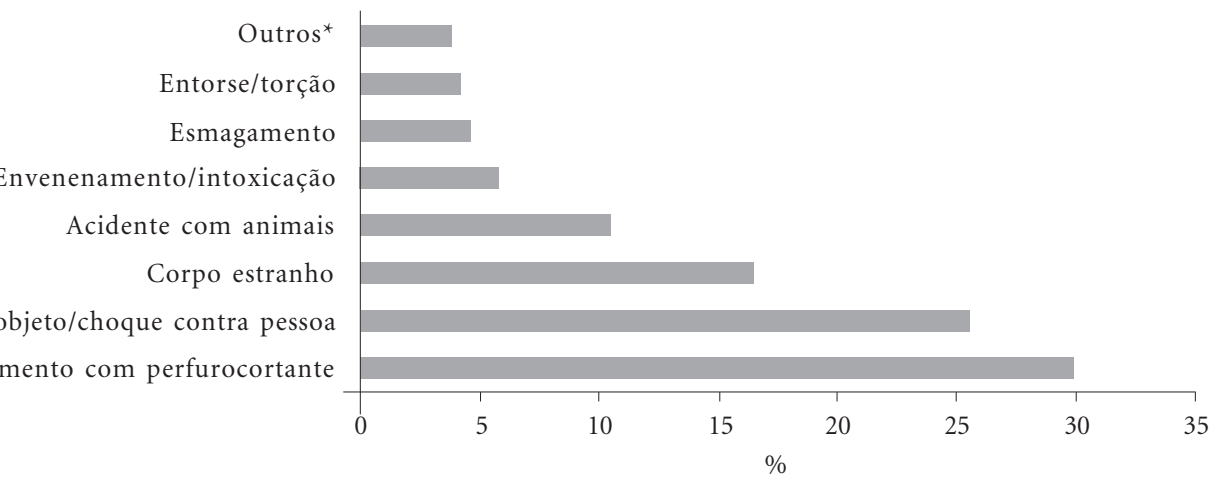

Figura 2. Distribuição proporcional de atendimentos de emergência por outras causas acidentais. Teresina-PI, 2009.

Fonte: MS, SVS, Sistema de Vigilância de Violências e Acidentes-VIVA, Inquérito 2009. *Inclui sufocação/engasgamento, afogamento, ferimento por arma de fogo. 
Na Tabela 1, constam algumas características dos atendimentos de emergência por outras causas acidentais segundo faixa etária das vítimas. Do total de atendimentos, $72,2 \%$ eram do sexo masculino e $27,8 \%$ do sexo feminino, não havendo diferença significativa desta distribuição entre os grupos de idade. Em relação ao quesito raça/cor da pele $83,6 \%$ dos indivíduos se autoidentificaram como negra, sendo proporcionalmente maior no grupo com idade de 20 e mais anos $(p=0,0084)$.
Não foi observada diferença significativa na distribuição dos atendimentos segundo tipo de ocorrência e faixa etária. A maioria dos eventos ocorreu no domicílio (54,6\%), principalmente para o grupo de menores de 20 anos $(61,5 \%)$, enquanto as ocorrências fora do domicílio foram significativamente mais frequentes entre os indivíduos de 20 e mais anos de idade (49,0\%; $\mathrm{p}=0,0318)$. A locomoção para o hospital se deu em $71,8 \%$ por veículo particular, $10,6 \%$ por assistência pré-hospitalar e $17,6 \%$ a pé/transporte

Tabela 1. Características dos atendimentos de emergência por outras causas acidentais segundo faixa etária. Teresina-PI 2009.

\begin{tabular}{|c|c|c|c|c|}
\hline \multirow[b]{2}{*}{ Características } & \multicolumn{3}{|c|}{ Faixa etária $(\text { anos })^{a}$} & \multirow[b]{2}{*}{ Valor de $\mathrm{p}^{\mathrm{b}}$} \\
\hline & $\begin{array}{c}\text { Total } \\
(\mathbf{n}=677) \\
\%\end{array}$ & $\begin{array}{c}<20 \\
(\mathbf{n}=239) \\
\%\end{array}$ & $\begin{array}{c}\geq 20 \\
(\mathrm{n}=438) \\
\%\end{array}$ & \\
\hline \multicolumn{5}{|l|}{ Sexo } \\
\hline Masculino & 72,2 & 72,0 & 72,4 & 0,9133 \\
\hline Feminino & 27,8 & 28,0 & 27,6 & \\
\hline \multicolumn{5}{|l|}{ Raça/cor da pele ${ }^{c}$} \\
\hline Branca & 16,4 & 21,7 & 13,5 & 0,0084 \\
\hline Negra (pretos+pardos) & 83,6 & 78,4 & 86,5 & \\
\hline \multicolumn{5}{|l|}{ Tipo de ocorrência } \\
\hline \multicolumn{5}{|l|}{ Ferimento com perfurocortante } \\
\hline Queda de objeto/choque contra pessoa & 29,8 & 28,5 & 30,6 & 0,6015 \\
\hline Corpo estranho & 25,4 & 28,0 & 24,0 & \\
\hline Acidente com animais & 16,4 & 14,2 & 17,6 & \\
\hline Outros $^{\mathrm{d}}$ & 10,3 & 11,3 & 9,8 & \\
\hline Local de ocorrência & 18,0 & 18,0 & 18,0 & \\
\hline Domicílio & 54,6 & 61,1 & 51,0 & 0,0318 \\
\hline Fora do domicílio & 45,4 & 38,9 & 49,0 & \\
\hline \multicolumn{5}{|l|}{ Locomoção para o hospital } \\
\hline Veículo particular & 71,8 & 67,8 & 74,1 & 0,1203 \\
\hline Assistência pré-hospitalar ${ }^{e}$ & 10,6 & 13,4 & 9,0 & \\
\hline A pé/transporte coletivo/outros ${ }^{\mathrm{f}}$ & 17,6 & 18,8 & 16,9 & \\
\hline \multicolumn{5}{|l|}{ Parte do corpo atingida } \\
\hline Tronco & 28,5 & 37,1 & 23,6 & 0,0010 \\
\hline Extremidades & 71,5 & 62,9 & 76,4 & \\
\hline \multicolumn{5}{|l|}{ Período do atendimento } \\
\hline Diurno (07h00min-18h59min) & 67,2 & 64,0 & 69,0 & 0,1931 \\
\hline Noturno (19h00min-06h59min) & 32,8 & 36,0 & 31,1 & \\
\hline \multicolumn{5}{|l|}{ Dia do atendimento } \\
\hline Sábado e domingo & 26,0 & 26,8 & 25,6 & 0,7513 \\
\hline Segunda a sexta & 74,0 & 73,2 & 74,4 & \\
\hline \multicolumn{5}{|l|}{ Evoluçãog } \\
\hline Alta & 84,1 & 78,5 & 87,2 & 0,0073 \\
\hline Internação/transferência para outro serviço & 15,9 & 21,6 & 12,8 & \\
\hline
\end{tabular}

Fonte: MS, SVS, Sistema de Vigilância de Violências e Acidentes-VIVA, Inquérito 2009. a) Para algumas variáveis o número de atendimentos divergiu devido a dados faltantes (ignorado/em branco). b) Teste do qui-quadrado. c) Excluídos registros com raça/cor da pele amarela $(n=14)$ e indígena $(n=1)$. d) Inclui sufocação/engasgamento, afogamento, envenenamento/intoxicação, ferimento por arma de fogo, entorse/torção, esmagamento, outros. e) Inclui unidades do Serviço de Atendimento Móvel de Urgência (SAMU), ambulâncias, resgate. f) Inclui viatura policial e outros. g) Excluídos os casos evadidos (n = 13) e que evoluíram ao óbito $(\mathrm{n}=2)$ nas primeiras 24 horas de atendimento. 
coletivo/outros, sem diferença entre os grupos de idade.

As extremidades (membros superiores e inferiores) foram as áreas anatômicas mais atingidas $(71,5 \%)$, sendo este segmento associado estatisticamente às ocorrências no grupo de $20 \mathrm{e}$ mais anos $(76,4 \%)$, enquanto as lesões no tronco (cabeça, tórax, abdome) apresentaram frequência mais elevada no grupo menor de 20 anos $(37,1 \% ; \mathrm{p}=0,0010)$. Para o período de atendimento, $67,2 \%$ ocorram no período diurno e $32,8 \%$ no noturno. Em relação ao dia de atendimento, $26,0 \%$ ocorreram no sábado e domingo e $74,0 \%$ de segunda a sexta-feira. Não houve diferença significativa entre os grupos de idade para o período e dia de atendimento. A evolução para alta foi o desfecho mais frequente $(84,1 \%)$, sendo maior no grupo de adultos e idosos (87,2\%) em relação aos mais jovens, dentre os quais a proporção de internação/transferência para outros serviços foi de 21,6\% ( $\mathrm{p}=0,0073)$.

A Tabela 2 apresenta as características dos atendimentos de emergência por outras causas acidentais segundo tipo de evento. Não houve diferença significativa entre as características da pessoa atendida (faixa etária, sexo, raça/cor da pele e escolaridade) segundo os tipos de evento. Independente do tipo de acidente, os atendimentos foram mais frequentes no grupo de $20 \mathrm{e}$ mais anos de idade, no sexo masculino, com raça/cor de pele negra e baixa escolaridade. Os atendimentos de emergência por corpo estranho e acidentes envolvendo animais foram significativamente mais frequentes no domicílio $(\mathrm{p}<0,05)$, enquanto queda de objeto/choque contra pessoa foram mais frequentes fora do domicílio $(\mathrm{p}=$ $0,0001)$.

Veículo particular foi o meio de locomoção mais utilizado para chegar ao hospital, sem verificação de diferenças significativas entre os diferentes tipos de acidente, exceto para os atendimentos por corpo estranho, nos quais a proporção de pacientes que chegaram a pé ou em transporte coletivo foi maior do que nos demais tipos de acidentes ( $p=0,0261)$.

Considerando somente os pacientes com idade a partir dos 20 anos, os acidentes com animais foram mais frequentes durante atividades não laborais $(81,4 \% ; \mathrm{p}=0,0032)$ em comparação aos demais tipos de evento. Para o mesmo grupo de pacientes, a declaração de consumo de bebida alcoólica foi maior entre as vítimas de acidentes com perfurocortantes $(9,7 \%$; $\mathrm{p}=$ $0,0220)$ em relação às vítimas dos demais tipos de acidente.
Em relação ao atendimento propriamente dito (dados sobre lesão e evolução), as lesões em extremidades foram mais frequentes para os atendimentos por ferimentos com perfurocortantes $(93 \% ; p=0,000)$, enquanto o tronco foi a parte do corpo mais frequentemente atingida para os atendimentos por queda de objeto/choque contra pessoa $(43,1 \% ; \mathrm{p}=0,0000)$ e aqueles envolvendo animais $(38,6 \% ; \mathrm{p}=0,0197)$ quando comparados aos atendimentos decorrentes das demais causas acidentais. Não houve diferença significativa entre as características do dia e período de atendimento e os tipos de acidente.

Quanto ao desfecho após o atendimento de urgência, houve maior proporção de alta dentre os atendimentos por ferimento perfurocortante $(90,3 \% ; \mathrm{p}=0,0272)$, enquanto a evolução para internação/transferência para outro serviço foi maior para os atendimentos por queda de objeto/choque contra pessoa $(19,4 \% ; \mathrm{p}=0,0042)$.

\section{Discussão}

O Sistema de Vigilância de Violências e Acidentes vem se consolidando como um sistema de informação que oferece, além das bases técnicas para ações específicas de prevenção e promoção da saúde, subsídios para que cada vez mais o modelo de vigilância em saúde se torne referência na organização do SUS. Ainda que sejam diversas as estratégias metodológicas utilizadas, e o VIVA Inquérito é uma delas, a principal motivação é informar a tomada de decisão ${ }^{28}$.

Nessa perspectiva, os resultados deste estudo evidenciaram que os hospitais municipais de referência em emergência para causas externas enfrentam, em sua maioria, a crescente demanda para atendimentos por outros acidentes. Essa situação é descrita em alguns estudos sobre atendimentos em hospitais de emergências, destacando-se o tipo de ocorrência e, consequentemente, a posição privilegiada em relação ao conjunto dos acidentes ${ }^{7-9,12,29,30}$.

As características relacionadas aos indivíduos mostraram diferença entre grupos de idade apenas para o quesito raça/cor da pele negra, maior para os indivíduos com idade a partir de 20 anos. O relatório do Inquérito VIVA 2007 e 2009 para o conjunto de 23 Capitais, Distrito Federal e municípios selecionados aponta que mais da metade dos atendimentos por outros acidentes, para todas as idades, ocorreu em pessoas negras ${ }^{1,12,21}$. Em Salvador, por exemplo, um estudo sobre mortalidade apresenta maior per- 
da de anos potenciais de vida e maior número médio de anos não vividos por causas externas entre as pessoas negras ${ }^{31}$.
No conjunto das características sobre o local de ocorrência, o domicílio foi identificado com maior proporção para crianças e adolescentes,

Tabela 2. Características dos atendimentos de emergência por outras causas acidentais segundo tipo de evento. Teresina-PI, 2009.

\begin{tabular}{|c|c|c|c|c|c|}
\hline \multirow[b]{2}{*}{ Características } & \multicolumn{5}{|c|}{ Tipo de evento ${ }^{a}$} \\
\hline & $\begin{array}{c}\text { Total }^{\mathrm{b}} \\
(\mathrm{n}=555) \\
\%\end{array}$ & $\begin{array}{c}\text { Ferimento } \\
\text { perfurocortante } \\
(\mathrm{n}=202) \\
\%\end{array}$ & $\begin{array}{c}\text { Queda de } \\
\text { objeto/choque } \\
\text { contra pessoa } \\
(\mathbf{n}=172) \\
\%\end{array}$ & $\begin{array}{c}\text { Corpo } \\
\text { estranho } \\
(\mathbf{n}=111) \\
\%\end{array}$ & $\begin{array}{c}\text { Acidentes } \\
\text { com } \\
\text { animais } \\
(\mathbf{n}=70) \\
\%\end{array}$ \\
\hline \multicolumn{6}{|l|}{ Faixa etária (em anos) } \\
\hline$<20$ & 35,3 & 33,7 & 39,0 & 30,6 & 38,6 \\
\hline$\geq 20$ & 64,7 & 66,3 & 61,1 & 69,4 & 61,4 \\
\hline \multicolumn{6}{|l|}{ Sexo } \\
\hline Masculino & 74,1 & 72,8 & 79,1 & 71,2 & 70,0 \\
\hline Feminino & 26,0 & 27,2 & 20,9 & 28,8 & 30,0 \\
\hline \multicolumn{6}{|l|}{ Raça/cor da pele ${ }^{c}$} \\
\hline Branca & 15,6 & 13,9 & 14,0 & 18,5 & 20,3 \\
\hline Negra (pretos+pardos) & 84,4 & 86,1 & 86,1 & 81,5 & 79,7 \\
\hline \multicolumn{6}{|l|}{ Escolaridade $^{\mathrm{d}}$} \\
\hline$\leq 8$ & 57,3 & 58,2 & 58,1 & 54,6 & 57,1 \\
\hline$\geq 9$ & 42,7 & 41,8 & 41,9 & 45,5 & 42,9 \\
\hline \multicolumn{6}{|l|}{ Local de ocorrência } \\
\hline Domicílio & 55,2 & 54,5 & 42,4 & $64,6^{\star}$ & $74,3^{\star}$ \\
\hline Fora do domicílio & 44,8 & 45,5 & $57,6^{*}$ & 35,5 & 25,7 \\
\hline \multicolumn{6}{|l|}{ Locomoção para o hospital } \\
\hline Veículo particular & 71,9 & 74,3 & 76,0 & 60,0 & 73,9 \\
\hline Assistência pré-hospitalare & 9,1 & 7,4 & 10,5 & 12,7 & $4,3^{\star *}$ \\
\hline A pé/transporte coletivo/outros ${ }^{\mathrm{f}}$ & 19,0 & 18,3 & 13,5 & $27,3^{\star}$ & 21,7 \\
\hline \multicolumn{6}{|l|}{ Evento relacionado ao trabalho ${ }^{d}$} \\
\hline $\operatorname{Sim}$ & 39,0 & 44,0 & 35,2 & 47,3 & 18,6 \\
\hline Não & 61,0 & 56,0 & 64,8 & 52,7 & $81,4^{*}$ \\
\hline \multicolumn{6}{|l|}{ Declaração de consumo de bebida alcoólica ${ }^{\mathrm{d}}$} \\
\hline Sim & 6,2 & $9,7^{*}$ & $3,8^{\star *}$ & $4,1^{\star \star}$ & $4,8^{\star *}$ \\
\hline Não & 93,8 & 90,3 & 96,2 & 95,9 & 95,2 \\
\hline \multicolumn{6}{|l|}{ Parte do corpo atingida } \\
\hline Tronco & 26,4 & 7,0 & $43,1^{*}$ & 38,9 & $38,6^{*}$ \\
\hline Extremidades & 73,6 & $93,0^{*}$ & 56,9 & 61,1 & 61,4 \\
\hline \multicolumn{6}{|l|}{ Evoluçãog } \\
\hline Alta & 86,1 & $90,3^{\star}$ & 80,6 & 90,7 & 80,9 \\
\hline Internação/transferência para outro serviço & 13,9 & 9,7 & $19,4^{*}$ & 9,3 & 19,1 \\
\hline \multicolumn{6}{|l|}{ Período do atendimento } \\
\hline Diurno (07h00min-18h59min) & 67,9 & 71,3 & 68,6 & 66,7 & 58,6 \\
\hline Noturno (19h00min-06h59min) & 32,1 & 28,7 & 31,4 & 33,3 & 41,4 \\
\hline \multicolumn{6}{|l|}{ Dia do atendimento } \\
\hline Sábado e domingo & 24,9 & 26,7 & 23,8 & 18,0 & 32,9 \\
\hline Segunda a sexta & 75,1 & 73,3 & 76,2 & 82,0 & 67,1 \\
\hline
\end{tabular}

Fonte: MS, SVS, Sistema de Vigilância de Violências e Acidentes-VIVA, Inquérito 2009. a) Para algumas variáveis o número de atendimentos divergiu devido a dados faltantes (ignorado/em branco). b) Excluídos os atendimentos por sufocação/engasgamento, afogamento, envenenamento/intoxicação, ferimento por arma de fogo, entorse/torção, esmagamento. c) Excluídos registros com raça/cor da pele amarela $(\mathrm{n}=14)$ e indígena $(\mathrm{n}=1)$. d) Inclui somente os casos com idade $\geq 20$ anos. e) Inclui unidades do Serviço de Atendimento Móvel de Urgência (SAMU), ambulâncias, resgate. f) Inclui viatura policial e outros. g) Excluídos os casos evadidos $(n=13)$ e que evoluíram ao óbito $(n=2)$ nas primeiras 24 horas de atendimento. ${ }^{*}$ Diferença estatisticamente significante entre o tipo de evento analisado e os demais tipos $(\mathrm{p}<0,05)$. ${ }^{* *}$ Não foram calculados qui-quadrado e valor de $\mathrm{p}$ devido a caselas com valor menor que cinco. 
semelhante a resultados encontrados para os anos de 2006 e 2007, para as Capitais, Distrito Federal e municípios selecionados ${ }^{1,7,10,21}$. Tal situação indica a necessidade de conhecer os determinantes e condicionantes que, guardando relação com ampla rede de fatores sociais, culturais, dos indivíduos e suas famílias, definem estilo de vida dos pais, condições do ambiente físico de convivência, do trabalho, além da socialização da criança e adolescente ${ }^{32}$.

Neste sentido, cuidados devem ser dispensados nesses espaços, especialmente nos lares pela presença de utensílios de cozinha, produtos e material de limpeza guardados em locais inapropriados, janelas sem proteção, escadas, animais domésticos, além de equipamentos e outros fatores de risco próprio do ambiente doméstico, que pela curiosidade e manuseio inapropriado, torna menores, idosos e indivíduos em geral vulneráveis a diversos acidentes, comumente chamados de acidentes domésticos ${ }^{7,32-36}$.

No grupo com idade de 20 anos e mais, o local de ocorrência de maior proporção foi fora do domicílio condizente com a idade produtiva, a dinâmica do mundo do trabalho e de suas vivências em diversos espaços e territórios. No entanto, para o idoso, há predomínio de ocorrência no domicílio, considerando os acidentes em geral, colocando novamente este ambiente como espaço de risco de acidentes em relação à idade. Este local pode trazer, para ambos os grupos, intercorrências traumáticas de importância variada, que vão desde escoriações a traumatismos mais severos $7,30,37$.

Não foi possível demonstrar qual o meio de locomoção para o hospital nem o tipo de ocorrência mais frequente entre os grupos de idade. Entretanto, sobre a locomoção para o hospital em busca de atendimento, a modalidade a pél transporte coletivo/outros foi mais frequente nos acidentes com penetração de corpo estranho.

Entre as crianças, alguns estudos apontam a penetração de corpo estranho em orifício natural, ingestão ou inalação, como sendo um dos cinco principais acidentes que ocorrem na população infantil ${ }^{33,38}$. Entre os adultos, estudo a partir do Inquérito VIVA 2009, apontam outros acidentes em segunda posição ${ }^{30}$.

Crianças e adolescentes tiveram o tronco como a parte do corpo mais atingida, semelhante a outros estudos ${ }^{8,10}$, enquanto os adultos e os idosos, em sua maioria, foram as extremidades. O desfecho evoluiu para alta nos atendimentos entre os indivíduos com 20 anos e mais, e nas crianças e adolescentes, a evolução, em sua mai- oria, foi para internação. Esses achados são semelhantes aos encontrados para o conjunto dos indivíduos participantes do Inquérito VIVA 2009, que traz, ainda em relação à natureza da lesão, as maiores proporções para corte/laceração, fraturas e contusão ${ }^{1}$.

As características da pessoa/vítima não apresentaram diferença segundo o tipo de evento. Mas, as relativas ao local de ocorrência evidenciam que no domicílio há maior proporção de ocorrência de penetração de corpo estranho e acidente com animais. Chama-se atenção para este espaço, pois nele se faz necessário o desenvolvimento de ações que abordem o cuidado com pequenos objetos como moedas, grãos/sementes, tampas e comportamentos provocativos que possam irritar animais, principalmente cães ${ }^{29,35,38}$. Diferentemente, para queda de objeto sobre pessoa/choque de pessoa contra objeto, a maior frequência foi observada fora do domicílio. Acredita-se que se pode incluir aqui, atividades de prática de lazer, esporte, trabalho e até via pública pela circulação de pessoas ${ }^{21}$.

Os acidentes com animais não foram considerados eventos relacionados ao trabalho, e nos demais não foi possível afirmar tal associação. No entanto, é vasta a literatura relacionando acidentes com instrumento perfurocortante ao trabalho, sobretudo, entre profissionais de saúde e empregadas domésticas ${ }^{6,11,39,40}$. Neste estudo, foi possível demonstrar a declaração de consumo de bebida alcoólica como característica para acidentes com perfurocortante, embora em outro, essa característica seja maior entre os acidentes de transporte ${ }^{41}$.

Com relação à localização da lesão, o tronco foi a parte do corpo mais atingida em acidentes do tipo queda de objeto sobre pessoa/choque de pessoa contra objeto e acidentes com animais. Já as extremidades foram partes mais atingidas nos acidentes com ferimentos provocados por instrumento perfurocortante. Resultados semelhantes foram encontrados no Inquérito VIVA 2007 para municípios selecionados e o Distrito Federal ${ }^{21}$.

O desfecho alta foi mais frequente nos atendimentos para ferimento com objeto perfurocortante, coincidente com os achados das edições anteriores do Inquérito VIVA no conjunto das capitais, que têm mostrado essa tendência em todos os anos de realização da pesquisa e em outros estudos ${ }^{1,10,12,21,30}$. Diferentemente, a evolução internação/transferência para outro serviço foi mais frequente para o evento queda de objeto sobre pessoa/choque de pessoas contra objeto, em relação aos demais, sem, no entanto, 
guardar semelhança junto aos resultados do Inquérito VIVA 2007 para este tipo de acidente ${ }^{21}$.

Por fim, apontar indisponibilidade de informações sobre outros acidentes que causam impacto no atendimento e reforçar a necessidade de conhecer melhor suas características, como observado no município de Teresina, são os aspectos inovadores que traz este artigo. Na perspectiva da efetividade de um sistema de vigilância e de causas externas tais elementos afirmam a importância, desafios e necessidades das unidades públicas de atendimento em emergência se tornarem fontes importantes para a obtenção de dados sobre esses eventos. Além disto, a magnitude dos acidentes como motivo de procura de atendimento sugere a necessidade de ampliação de programas e projetos que possam incluir as-

\section{Referências}

1. Brasil. Ministério da Saúde (MS). Departamento de Análise de Situação de Saúde. VIVA: vigilância de violências e acidentes, 2008 e 2009. Brasília: MS; 2010.

2. Brasil. Ministério da Saúde. Portaria GM/MS n. 737, de 16 de maio de 2001. Institui a política nacional de redução da morbi-mortalidade por acidentes e violências. Diário Oficial da União 2001; 18 nov.

3. Minayo MCS. Violência: um problema para a saúde dos brasileiros. In: Brasil. Ministério da Saúde (MS). Impacto da violência na saúde dos brasileiros. Brasília: (MS); 2005. p. 9-42.

4. Mello Jorge MHP, Laurenti R. Acidentes e violência no Brasil - Apresentação. Rev Saude Publica 1997; 31(Supl. 4):1-4.

5. Unglert CVS, Siqueira AAF, Carvalho GA. Características epidemiológicas dos acidentes na infância. Rev Saude Publica 1987; 21(3):234-245.

6. Moura JP, Gir E, Canini SRMS. Acidentes ocupacionais com material perfurocortante em um hospital regional de Minas Gerais, Brasil. Ciencia y enfermeria 2006; XII(1):29-37.

7. Gawryszewski VP, Scarpelini S, Dib JA, Mello Jorge MHP, Pereira Junior GA, Morita M. Atendimentos de emergência por lesões decorrentes de causas externas: características das vítimas e local de ocorrência, Estado de São Paulo, Brasil, 2005. Cad Saude Publica 2008; 24(5):1121-1129.

8. Martins CBG, Andrade SM. Causas externas entre menores de 15 anos em cidade do Sul do Brasil: atendimentos em pronto socorro, internações e óbitos. Rev Bras Epidemiol 2005; 8(2):194-204.

9. Deslandes SF. O atendimento às vítimas de violência na emergência: prevenção numa hora dessas? Cien Saude Colet 1999; 4(1):81-94. pectos de redução de vulnerabilidades aos acidentes, ações de prevenção e promoção da saúde, articulando ações a serem desenvolvidas nos serviços, nos domicílios, escolas e espaços comunitários.

\section{Colaboradores}

AAG Pedrosa, MDM Mascarenhas, EM Costa e LP Cronemberger participaram igualmente da concepção, delineamento, análise e interpretação dos dados, redação e revisão crítica do artigo.
10. Malta DC, Mascarenhas MDM, Silva MMA, Macário EM. Perfil dos atendimentos de emergência por acidentes envolvendo crianças menores de dez anos - Brasil, 2006 a 2007. Cien Saude Colet 2009; 14(5): 1669-1679.

11. Canini SRMS, Gir E, Hayashida M, Machado AA. Acidentes perfurocortantes entre trabalhadores de enfermagem de um hospital universitário do interior paulista. Rev Latino-am Enfermagem 2002; 10(2):172-178.

12. Mascarenhas MDM, Silva MMA, Malta DC, Moura L, Gawryszewski VP, Costa VC, Souza MFM, Morais Neto OL. Atendimentos de emergência por acidentes na Rede de Vigilância de Violências e Acidentes - Brasil, 2006. Cien Saude Colet 2009; 14(5): 1657-1668.

13. Carvalho MFPP, Puccini RF, Silva EMK. Acidentes não fatais em adolescentes escolares de Belém, Pará. Rev Paul Pediatr 2007; 25(4):324-330.

14. Macedo JLS, Camargo LM, Almeida PF, Rosa SC. Perfil epidemiológico do trauma de face dos pacientes atendidos no pronto socorro de um hospital público. Rev Col Bras Cir 2008; 35(1):9-13.

15. Vasconcellos MM, Gribel EB, Moraes IHS. Registros em saúde: avaliação da qualidade do prontuário do paciente na atenção básica, Rio de Janeiro, Brasil. Cad Saude Publica 2008; 24(Supl. 1): S173-S182.

16. Melione LPR, Mello Jorge MHP. Gastos do Sistema Único de Saúde com internações por causas externas em São José dos Campos, São Paulo, Brasil. Cad Saude Publica 2008; 24(8):1814-1824.

17. Tomimatsu MFAI, Andrade SM, Soares DA, Mathias TAF, Sapata MPM, Soares DFPP, Souza RKT. Qualidade da informação sobre causas externas no Sistema de Informações Hospitalares. Rev Saude Publica 2009; 43(3):413-420. 
18. Minayo MCS, Deslandes SF. Análise da implantação da rede de atenção às vítimas de acidentes e violências segundo diretrizes da Política Nacional de Redução da Morbimortalidade sobre Violência e Saúde. Cien Saude Colet 2009; 14(5):1641-1649.

19. Gawryszewski VP, Silva MMA, Malta DC, Mascarenhas MDM, Costa VC, Matos SG, Morais Neto OL, Monteiro RA, Carvalho CG, Magalhães ML. A proposta da rede de serviços sentinela como estratégia de vigilância de violências e acidentes. Cien Saude Colet 2007; 11(Supl.):1269-1278.

20. Malta DC, Lemos MSA, Silva MMA, Rodrigues MES, Gazal-Carvalho C, Morais Neto OL. Iniciativas de vigilância e prevenção de acidentes e violências no contexto do Sistema Único de Saúde (SUS). Epidemiol Serv Saúde 2007; 16(1):45-55.

21. Brasil. Ministério da Saúde (MS). VIVA: vigilância de violências e acidentes, 2006 e 2007. Brasília: MS; 2009.

22. Malta DC, Silva MMA, Mascarenhas MDM, Sá NNB, Morais Neto OL, Bernal RTI, Monteiro RA, Andrade SSCA, Gawryszewski VP. Características e fatores associados às quedas atendidas em serviços de emergência. Rev Saude Publica 2012; 46(1):128-137.

23. Malta DC, Bernal RTI, Mascarenhas MDM, Monteiro RA, Sá NNB, Andrade SSCA, Gawryszewski VP, Silva MMA, Morais Neto OL. Atendimentos por acidentes de transporte em serviços públicos de emergência em 23 capitais e no Distrito Federal - Brasil, 2009. Epidemiol. Serv. Saúde 2012; 21(1):31-42.

24. Gawryszewski VP, Bernal RTI, Silva NN, Morais Neto OL, Silva MMA, Mascarenhas MDM, Sá NNB, Monteiro RA, Malta DC. Atendimentos decorrentes de queimaduras em serviços públicos de emergência no Brasil, 2009. Cad Saude Publica 2012; 28(4): 629-640.

25. Brasil. Ministério da Saúde (MS). Inquérito sobre Atendimentos por Violências e Acidentes em Serviços Sentinela de Urgência e Emergência do Sistema de Vigilância de Violências e Acidentes (VIVA) Capitais, Distrito Federal e municípios selecionados - Manual do Entrevistador (impresso). Brasília: MS; 2011.

26. Organização Mundial de Saúde (OMS). Classificação estatística internacional de doenças e problemas relacionados à saúde. Décima revisão (CID-10), v. 1. São Paulo: ENDUSP; 2009.

27. Brasil. Ministério da Saúde (MS). Conselho Nacional de Saúde. Resolução no. 196/1996. Diretrizes e Normas Regulamentadoras de Pesquisas Envolvendo Seres Humanos. Diário Oficial da União 1996; out 10.

28. Brasil. Ministério da Saúde (MS). Saúde Brasil 2010: uma análise da situação de saúde e de evidências selecionadas de impacto de ações de vigilância em saúde. Brasília: MS; 2011.

29. Mesquita Filho M, Mello Jorge MHP. Características da morbidade por causas externas em serviço de urgência. Rev Bras Epidemiol 2007; 10(4):679-691.

30. Luz TCB, Malta DC, Sá NNB, Silva MMA, LimaCosta MF. Violências e acidentes entre adultos mais velhos em comparação aos mais jovens: evidências do Sistema de Vigilância de Violências e Acidentes (VIVA), Brasil. Cad Saude Publica 2011; 27(11):21352142.
31. Araújo EM, Costa MCN, Hogan VK, Mota ELA, Araújo TM, Oliveira NF. Diferenciais de raça/cor da pele em anos potenciais de vida perdidos por causas externas. Rev Saude Publica 2009; 43(3):405-412.

32. Martins CBG. Acidentes na infância e adolescência: uma revisão bibliográfica. Rev Bras Enferm 2006; 59(3):344-348.

33. Filócomo FRF, Harada MJCS, Silva CV, Pedreira MLG. Estudo dos acidentes na infância em um pronto socorro pediátrico. Rev Latino-am Enfermagem 2002; 10(1):41-47.

34. Souza LJEX, Rodrigues AKC, Barros MGT. A família vivenciando o acidente doméstico - relato de uma experiência. Rev Latino-am Enferm 2000; 8(1): 83-89.

35. Ciampo LAD, Riccoa RG, Almeida CAN, Martins LRCB, Santos TCC. Acidentes de mordeduras de cães na infância. Rev Saude Publica 2000; 34(4):411412.

36. Vieira LJES, Carneiro RCMM, Frota MA, Gomes ALA, Ximenes LB. Ações e possibilidades de prevenção de acidentes com crianças em creches de Fortaleza, Ceará. Cien Saude Colet 2009; 14(5):16871697.

37. Lima RS, Campos MLP. Perfil do idoso vítima de trauma atendido em uma Unidade de Urgência e Emergência. Rev Esc Enferm USP 2011; 45(3):659664.

38. Martins CBG, Andrade, SM. Acidentes com corpo estranho em menores de 15 anos: análise epidemiológica dos atendimentos em pronto-socorro, internações e óbitos. Cad Saude Publica 2008; 24(9):19831990.

39. Santana VS, Amorim AM, Xavier S, Oliveira R, Xavier S, Iriart J, Belitardo L. Emprego em serviços domésticos e acidentes de trabalho não fatais. Rev Saude Publica 2003; 37(1):65-74.

40. Marziale MHP, Rodrigues CM. A produção científica sobre os acidentes de trabalho com material perfurocortante entre trabalhadores de enfermagem. Rev Latino-am Enferm 2002; 10(4):571-577.

41. Mascarenhas MDM, Malta DC, Silva MMA, Carvalho CG, Monteiro RA, Morais Neto OL. Consumo de álcool entre vítimas de acidentes e violências atendidas em serviços de emergência no Brasil, 2006 e 2007. Cien Saude Colet 2009; 14(5):1789-1796.

Artigo apresentado em 10/06/2012

Aprovado em 04/07/2012

Versão final apresentada em 14/07/2012 